UH-IfA-94/35

SU-ITP-94-13

YITP/U-94-15

hep-th/9405187

\title{
REHEATING AFTER INFLATION
}

\author{
Lev Kofman \\ Institute for Astronomy, University of Hawaii, 2680 Woodlawn Dr., Honolulu, HI 96822, USA] \\ Andrei Linde \\ Department of Physics, Stanford University, Stanford, CA 94305, USA 7 \\ and
}

Alexei A. Starobinsky

Yukawa Institute for Theoretical Physics, Kyoto University, Uji 611, Japan

and Landau Institute for Theoretical Physics, Kosygina St. 2, Moscow 117334, Russia

\begin{abstract}
The theory of reheating of the Universe after inflation is developed. We have found that typically at the first stage of reheating the classical inflaton field $\phi$ rapidly decays into $\phi$-particles or into other bosons due to a broad parametric resonance. Then these bosons decay into other particles, which eventually become thermalized. Complete reheating is possible only in those theories where a single particle $\phi$ can decay into other particles. This imposes strong constraints on the structure of inflationary models, and implies that the inflaton field can be a dark matter candidate.
\end{abstract}

PACS numbers: $98.80 . \mathrm{Cq}, 04.62 .+\mathrm{v}$

\footnotetext{
${ }^{1}$ On leave of absence from Institute of Astrophysics and Atmospheric Physics, Tartu EE-2444, Estonia

${ }^{2}$ On leave of absence from Lebedev Physical Institute, Moscow 117924, Russia
} 
1. The theory of reheating of the Universe after inflation is the most important application of the quantum theory of particle creation, since almost all matter constituting the Universe at the subsequent radiation-dominated stage was created during this process [1]. At the stage of inflation all energy was concentrated in a classical slowly moving inflaton field $\phi$. Soon after the end of inflation this field began to oscillate near the minimum of its effective potential. Gradually it produced many elementary particles, they interacted with each other and came to a state of thermal equilibrium with some temperature $T_{r}$, which was called the reheating temperature.

An elementary theory of reheating was first developed in [2] for the new inflationary scenario. Independently a theory of reheating in the $R^{2}$ inflation was constructed in [3]. Various aspects of this theory were further elaborated by many authors, see e.g. [4]. Still, a general scenario of reheating was absent. In particular, reheating in the chaotic inflation theory remained almost unexplored. The present paper is a short account of our investigation of this question [5]. We have found that the process of reheating typically consists of three different stages. At the first stage, which cannot be described by the elementary theory of reheating, the classical coherently oscillating inflaton field $\phi$ decays into massive bosons (in particular, into $\phi$-particles) due to parametric resonance. In many models the resonance is very broad, and the process occurs extremely rapidly (explosively). Because of the Pauli exclusion principle, there is no explosive creation of fermions. To distinguish this stage from the stage of particle decay and thermalization, we will call it pre-heating. Bosons produced at that stage are far away from thermal equilibrium and typically have enormously large occupation numbers. The second stage is the decay of previously produced particles. This stage typically can be described by methods developed in [2]. However, these methods should be applied not to the decay of the original homogeneous inflaton field, but to the decay of particles and fields produced at the stage of explosive reheating. This considerably changes many features of the process, including the final value of the reheating temperature. The third stage is the stage of thermalization, which can be described by standard methods, see e.g. 11, 2n; we will not consider it here. Sometimes this stage may occur simultaneously with the second one. In our investigation we have used the formalism of the time-dependent Bogoliubov transformations to find the density of created particles, $n_{\vec{k}}(t)$. A detailed description of this theory will be given in [5]; here we will outline our main conclusions using a simple semiclassical approach.

2. We will consider a simple chaotic inflation scenario describing the classical inflaton scalar field $\phi$ with the effective potential $V(\phi)= \pm \frac{1}{2} m_{\phi}^{2} \phi^{2}+\frac{\lambda}{4} \phi^{4}$. Minus sign corresponds to spontaneous symmetry breaking $\phi \rightarrow \phi+\sigma$ with generation of a classical scalar field $\sigma=\frac{m_{\phi}}{\sqrt{\lambda}}$. The field $\phi$ after inflation may decay into bosons $\chi$ and fermions $\psi$ due to the interaction terms $-\frac{1}{2} g^{2} \phi^{2} \chi^{2}$ and $-h \bar{\psi} \psi \phi$. Here $\lambda, g$ and $h$ are small coupling constants. In case of spontaneous symmetry breaking, the term $-\frac{1}{2} g^{2} \phi^{2} \chi^{2}$ gives rise to the term $-g^{2} \sigma \phi \chi^{2}$. We will assume for simplicity that the bare masses of the fields $\chi$ and $\psi$ are very small, so that one can write $m_{\chi}(\phi)=g \phi, m_{\psi}(\phi)=|h \phi|$.

Let us briefly recall the elementary theory of reheating [1]. At $\phi>M_{p}$, we have a stage of inflation. This stage is supported by the friction-like term $3 H \dot{\phi}$ in the equation of motion for the scalar field. Here $H \equiv \dot{a} / a$ is the Hubble parameter, $a(t)$ is the scale factor of the Universe. However, with a decrease of the field $\phi$ this term becomes less and less important, and inflation ends at $\phi \lesssim M_{p} / 2$. After that the field $\phi$ begins oscillating near the minimum of $V(\phi)$ [6]. The amplitude 
of the oscillations gradually decreases because of expansion of the universe, and also because of the energy transfer to particles created by the oscillating field. Elementary theory of reheating is based on the assumption that the classical oscillating scalar field $\phi(t)$ can be represented as a collection of scalar particles at rest. Then the rate of decrease of the energy of oscillations coincides with the decay rate of $\phi$-particles. The rates of the processes $\phi \rightarrow \chi \chi$ and $\phi \rightarrow \psi \psi\left(\right.$ for $m_{\phi} \gg 2 m_{\chi}, 2 m_{\psi}$ ) are given by

$$
\Gamma(\phi \rightarrow \chi \chi)=\frac{g^{4} \sigma^{2}}{8 \pi m_{\phi}}, \quad \Gamma(\phi \rightarrow \psi \psi)=\frac{h^{2} m_{\phi}}{8 \pi} .
$$

Reheating completes when the rate of expansion of the universe given by the Hubble constant $H=\sqrt{\frac{8 \pi \rho}{3 M_{p}^{2}}} \sim t^{-1}$ becomes smaller than the total decay rate $\Gamma=\Gamma(\phi \rightarrow \chi \chi)+\Gamma(\phi \rightarrow \psi \psi)$. The reheating temperature can be estimated by $T_{r} \simeq 0.1 \sqrt{\Gamma M_{p}}$.

As we already mentioned, this theory can provide a qualitatively correct description of particle decay at the last stages of reheating. Moreover, this theory is always applicable if the inflaton field can decay into fermions only, with a small coupling constant $h^{2} \ll m_{\phi} / M_{p}$. However, typically this theory is inapplicable to the description of the first stages of reheating, which makes the whole process quite different. In what follows we will develop the theory of the first stages of reheating. We will begin with the theory of a massive scalar field $\phi$ decaying into particles $\chi$, then we consider the theory $\frac{\lambda}{4} \phi^{4}$, and finally we will discuss reheating in the theories with spontaneous symmetry breaking.

3. We begin with the investigation of the simplest inflationary model with the effective potential $\frac{m_{\phi}^{2}}{2} \phi^{2}$. Suppose that this field only interacts with a light scalar field $\chi\left(m_{\chi} \ll m_{\phi}\right)$ due to the term $-\frac{1}{2} g^{2} \phi^{2} \chi^{2}$. The equation for quantum fluctuations of the field $\chi$ with the physical momentum $\vec{k} / a(t)$ has the following form:

$$
\ddot{\chi}_{k}+3 H \dot{\chi}_{k}+\left(\frac{k^{2}}{a^{2}(t)}+g^{2} \Phi^{2} \sin ^{2}\left(m_{\phi} t\right)\right) \chi_{k}=0,
$$

where $k=\sqrt{\vec{k}^{2}}$, and $\Phi$ stands for the amplitude of oscillations of the field $\phi$. As we shall see, the main contribution to $\chi$-particle production is given by excitations of the field $\chi$ with $k / a \gg m_{\phi}$, which is much greater than $H$ at the stage of oscillations. Therefore, in the first approximation we may neglect the expansion of the Universe, taking $a(t)$ as a constant and omitting the term $3 H \dot{\chi}_{k}$ in (2). Then the equation (2) describes an oscillator with a variable frequency $\Omega_{k}^{2}(t)=$ $k^{2} a^{-2}+g^{2} \Phi^{2} \sin ^{2}\left(m_{\phi} t\right)$. Particle production occurs due to a nonadiabatic change of this frequency. Equation (2) can be reduced to the well-known Mathieu equation:

$$
\chi_{k}^{\prime \prime}+(A(k)-2 q \cos 2 z) \chi_{k}=0
$$

where $A(k)=\frac{k^{2}}{m_{\phi}^{2} a^{2}}+2 q, q=\frac{g^{2} \Phi^{2}}{4 m_{\phi}^{2}}, z=m_{\phi} t$, prime denotes differentiation with respect to $z$. An important property of solutions of the equation (3) is the existence of an exponential instability $\chi_{k} \propto \exp \left(\mu_{k}^{(n)} z\right)$ within the set of resonance bands of frequencies $\Delta k^{(n)}$ labeled by an integer index $n$. This instability corresponds to exponential growth of occupation numbers of quantum 
fluctuations $n_{\vec{k}}(t) \propto \exp \left(2 \mu_{k}^{(n)} m_{\phi} t\right)$ that may be interpreted as particle production. The simplest way to analyse this effect is to study the stability/instability chart of the Mathieu equation, which is sketched in Fig. 1. White bands on this chart correspond to the regions of instability, the grey bands correspond to regions of stability. The curved lines inside white bands show the values of the instability parameter $\mu_{k}$. The line $A=2 q$ shows the values of $A$ and $q$ for $k=0$. All points in the white regions above this line correspond to instability for any given $q$. As one can see, near the line $A=2 q$ there are regions in the first, the second and the higher instability bands where the unstable modes grow extremely rapidly, with $\mu_{k} \sim 0.2$. We will show analytically in [5] that for $q \gg 1$ typically $\mu_{k} \sim \frac{\ln 3}{2 \pi} \approx 0.175$ in the instability bands along the line $A=2 q$, but its maximal value is $\frac{\ln (1+\sqrt{2})}{\pi} \approx 0.28$. Creation of particles in the regime of a broad resonance $(q>1)$ with $2 \pi \mu_{k}=O(1)$ is very different from that in the usually considered case of a narrow resonance $(q \ll 1)$, where $2 \pi \mu_{k} \ll 1$. In particular, it proceeds during a tiny part of each oscillation of the field $\phi$ when $1-\cos z \sim q^{-1}$ and the induced effective mass of the field $\chi$ (which is determined by the condition $m_{\chi}^{2}=g^{2} \Phi^{2} / 2$ ) is less than $m_{\phi}$. As a result, the number of particles grows exponentially within just a few oscillations of the field $\phi$. This leads to an extremely rapid (explosive) decay of the classical scalar field $\phi$. This regime occurs only if $q \gtrsim \pi^{-1}$, i.e. for $g \Phi \gtrsim m_{\phi}$, so that $m_{\phi} \ll g M_{p}$ is the necessary condition for it. One can show that a typical energy $E$ of a particle produced at this stage is determined by equation $A-2 q \sim \sqrt{q}$, and is given by $E \sim \sqrt{g m_{\phi} M_{p}}[5]$.

Creation of $\chi$-particles leads to the two main effects: transfer of the energy from the homogeneous field $\phi(t)$ to these particles and generation of the contribution to the effective mass of the $\phi$ field: $m_{\phi, e f f}^{2}=m_{\phi}^{2}+g^{2}\left\langle\chi^{2}\right\rangle_{r e n}$. The last term in the latter expression quickly becomes larger than $m_{\phi}^{2}$. One should take both these effects into account when calculating backreaction of created particles on the process. As a result, the stage of the broad resonance creation ends up within the short time $t \sim m_{\phi}^{-1} \ln \left(m_{\phi} / g^{5} M_{p}\right)$, when $\Phi^{2} \sim\left\langle\chi^{2}\right\rangle$ and $q=\frac{g^{2} \Phi^{2}}{4 m_{\phi, e f f}^{2}}$ becomes smaller than 1. At this time the energy density of produced particles $\sim E^{2}\left\langle\chi^{2}\right\rangle \sim g m_{\phi} M_{p} \Phi^{2}$ is of the same order as the original energy density $\sim m_{\phi}^{2} M_{p}^{2}$ of the scalar field $\phi$ at the end of inflation. This gives the amplitude of oscillations at the end of the stage of the broad resonance particle creation: $\Phi^{2} \sim\left\langle\chi^{2}\right\rangle \sim g^{-1} m_{\phi} M_{p} \ll M_{p}^{2}$. Since $E \gg m_{\phi}$, the effective equation of state of the whole system becomes $p \approx \varepsilon / 3$. Thus, explosive creation practically eliminates a prolonged intermediate matter-dominated stage after the end of inflation which was thought to be characteristic to many inflationary models. However, this does not mean that the process of reheating has been completed. Instead of $\chi$-particles in the thermal equilibrium with a typical energy $E \sim T \sim\left(m M_{p}\right)^{1 / 2}$, one has particles with a much smaller energy $\sim\left(g m_{\phi} M_{p}\right)^{1 / 2}$, but with extremely large mean occupation numbers $n_{k} \sim g^{-2} \gg 1$.

After that the Universe expands as $a(t) \propto \sqrt{t}$, and the scalar field $\phi$ continues its decay in the regime of the narrow resonance creation $q \approx \frac{\Phi^{2}}{4\left\langle\chi^{2}\right\rangle} \ll 1$. As a result, $\phi$ decreases rather slowly, $\phi \propto t^{-3 / 4}$. This regime is very important because it makes the energy of the $\phi$ field much smaller than that of the $\chi$-particles. One can show that the decay finally stops when the amplitude of oscillations $\Phi$ becomes smaller than $g^{-1} m_{\phi}[5]$. This happens at the moment $t \sim m_{\phi}^{-1}\left(g M_{p} / m_{\phi}\right)^{1 / 3}$ (in the case $m<g^{7} M_{p}$ decay ends somewhat later, in the perturbative regime). The physical reason why the decay stops is rather general: decay of the particles $\phi$ in our model occurs due to its interaction with another $\phi$-particle (interaction term is quadratic in $\phi$ and in $\chi$ ). When the field $\phi$ 
(or the number of $\phi$-particles) becomes small, this process is inefficient. The scalar field can decay completely only if a single scalar $\phi$-particle can decay into other particles, due to the processes $\phi \rightarrow \chi \chi$ or $\phi \rightarrow \psi \psi$, see eq. (1). If there is no spontaneous symmetry breaking and no interactions with fermions in our model, such processes are impossible.

At later stages the energy of oscillations of the inflaton field decreases as $a^{-3}(t)$, i.e. more slowly than the decrease of energy of hot ultrarelativistic matter $\propto a^{-4}(t)$. Therefore, the relative contribution of the field $\phi(t)$ to the total energy density of the Universe rapidly grows. This gives rise to an unexpected possibility that the inflaton field by itself, or other scalar fields can be cold dark matter candidates, even if they strongly interact with each other. However, this possibility requires a certain degree of fine tuning; a more immediate application of our result is that it allows one to rule out a wide class of inflationary models which do not contain interaction terms of the type of $g^{2} \sigma \phi \chi^{2}$ or $h \phi \bar{\psi} \psi$.

4. So far we have not considered the term $\frac{\lambda}{4} \phi^{4}$ in the effective potential. Meanwhile this term leads to production of $\phi$-particles, which in some cases appears to be the leading effect. Let us study the $\phi$-particle production in the theory with $V(\phi)=\frac{m_{\phi}^{2}}{2} \phi^{2}+\frac{\lambda}{4} \phi^{4}$ with $m_{\phi}^{2} \ll \lambda M_{p}^{2}$. In this case the effective potential of the field $\phi$ soon after the end of inflation at $\phi \sim M_{p}$ is dominated by the term $\frac{\lambda}{4} \phi^{4}$. Oscillations of the field $\phi$ in this theory are not sinusoidal, they are given by elliptic functions, but with a good accuracy one can write $\phi(t) \sim \Phi \sin \left(c \sqrt{\lambda} \int \Phi d t\right)$, where $c=\frac{\Gamma^{2}(3 / 4)}{\sqrt{\pi}} \approx 0.85$. The Universe at that time expands as at the radiation-dominated stage: $a(t) \propto \sqrt{t}$. If one neglects the feedback of created $\phi$-particles on the homogeneous field $\phi(t)$, then its amplitude $\Phi(t) \propto a^{-1}(t)$, so that $a \Phi=$ const. Using a conformal time $\eta$, exact equation for quantum fluctuations $\delta \phi$ of the field $\phi$ can be reduced to the Lame equation. The results remain essentially the same if we use an approximate equation

$$
\frac{d^{2}\left(\delta \phi_{k}\right)}{d \eta^{2}}+\left[k^{2}+3 \lambda a^{2} \Phi^{2} \sin ^{2}(c \sqrt{\lambda} a \Phi \eta)\right] \delta \phi_{k}=0, \quad \eta=\int \frac{d t}{a(t)}=\frac{2 t}{a(t)},
$$

which leads to the Mathieu equation with $A=\frac{k^{2}}{c^{2} \lambda a^{2} \Phi^{2}}+\frac{3}{2 c^{2}} \approx \frac{k^{2}}{c^{2} \lambda a^{2} \Phi^{2}}+2.08$, and $q=\frac{3}{4 c^{2}} \approx 1.04$. Looking at the instability chart, we see that the resonance occurs in the second band, for $k^{2} \sim$ $3 \lambda a^{2} \Phi^{2}$. The maximal value of the coefficient $\mu_{k}$ in this band for $q \sim 1$ approximately equals to 0.07. As long as the backreaction of created particles is small, expansion of the Universe does not shift fluctuations away from the resonance band, and the number of produced particles grows as $\exp \left(2 c \mu_{k} \sqrt{\lambda} a \Phi \eta\right) \sim \exp \left(\frac{\sqrt{\lambda} \Phi t}{5}\right)$.

After the time interval $\sim M_{p}^{-1} \lambda^{-1 / 2}|\ln \lambda|$, backreaction of created particles becomes significant. The growth of the fluctuations $\left\langle\phi^{2}\right\rangle$ gives rise to a contribution $3 \lambda\left\langle\phi^{2}\right\rangle$ to the effective mass squared of the field $\phi$, both in the equation for $\phi(t)$ and in Eq. (四) for inhomogeneous modes. The stage of explosive reheating ends when $\left\langle\phi^{2}\right\rangle$ becomes greater than $\Phi^{2}$. After that, $\Phi^{2} \ll\left\langle\phi^{2}\right\rangle$ and the effective frequency of oscillations is determined by the term $\sqrt{3 \lambda\left\langle\phi^{2}\right\rangle}$. The corresponding process is described by Eq. (4) with $A(k)=1+2 q+\frac{k^{2}}{3 \lambda a^{2}\left\langle\phi^{2}\right\rangle}, q=\frac{\Phi^{2}}{4\left\langle\phi^{2}\right\rangle}$. In this regime $q \ll 1$, and particle creation occurs in the narrow resonance regime in the second band with $A \approx 4$. Decay of the field in this regime is extremely slow: the amplitude $\Phi$ decreases only by a factor $t^{1 / 12}$ faster that it 
would decrease without any decay, due to the expansion of the Universe only, i.e., $\Phi \propto t^{-7 / 12}$ [5]. Reheating stops altogether when the presence of non-zero mass $m_{\phi}$ though still small as compared to $\sqrt{3 \lambda\left\langle\phi^{2}\right\rangle}$ appears enough for the expansion of the Universe to drive a mode away from the narrow resonance. It happens when the amplitude $\Phi$ drops up to a value $\sim m_{\phi} / \sqrt{\lambda}$.

In addition to this process, the field $\phi$ may decay to $\chi$-particles. This is the leading process for $g^{2} \gg \lambda$. The equation for $\chi_{k}$ quanta has the same form as eq. (雨) with the obvious change $\lambda \rightarrow g^{2} / 3$. Initially parametric resonance is broad. The values of the parameter $\mu_{k}$ along the line $A=2 q$ do not change monotonically, but typically for $q \gg 1$ they are 3 to 4 times greater than the parameter $\mu_{k}$ for the decay of the field $\phi$ into its own quanta. Therefore, this pre-heating process is very efficient. It ends at the moment $t \sim M_{p}^{-1} \lambda^{-1 / 2} \ln \left(\lambda / g^{10}\right)$ when $\Phi^{2} \sim\left\langle\chi^{2}\right\rangle \sim g^{-1} \sqrt{\lambda} M_{p}^{2}$. The typical energy of created $\chi$-particles is $E \sim\left(g^{2} \lambda\right)^{1 / 4} M_{p}$. The following evolution is essentially the same as that described in Sec. 3 .

5. Finally, let us consider the case with symmetry breaking. In the beginning, when the amplitude of oscillations is much greater than $\sigma$, the theory of decay of the inflaton field is the same as in the case considered above. The most important part of pre-heating occurs at this stage. When the amplitude of the oscillations becomes smaller than $m_{\phi} / \sqrt{\lambda}$ and the field begins oscillating near the minimum of the effective potential at $\phi=\sigma$, particle production due to the narrow parametric resonance typically becomes very weak. The main reason for this is related to the backreaction of particles created at the preceding stage of pre-heating on the rate of expansion of the universe and on the shape of the effective potential [5]. However, importance of spontaneous symmetry breaking for the theory of reheating should not be underestimated, since it gives rise to the interaction term $g^{2} \sigma \phi \chi^{2}$ which is linear in $\phi$. Such terms are necessary for a complete decay of the inflaton field in accordance with the perturbation theory (11).

6. In this paper we discussed the process of reheating of the universe in various inflationary models. We have found that decay of the inflaton field typically begins with a stage of explosive production of particles at a stage of a broad parametric resonance. Later the resonance becomes narrow, and finally this stage of decay finishes altogether. Interactions of particles produced at this stage, their decay into other particles and subsequent thermalization typically require much more time that the stage of pre-heating, since these processes are suppressed by the small values of coupling constants. The corresponding processes in many cases can be described by the elementary theory of reheating. However, this theory should be applied not to the decay of the original large and homogeneous oscillating inflaton field, but to the decay of particles produced at the stage of pre-heating, as well as to the decay of small remnants of the classical inflaton field. This makes a lot of difference, since typically coupling constants of interaction of the inflaton field with matter are extremely small, whereas coupling constants involved in the decay of other bosons can be much greater. As a result, the reheating temperature can be much higher than the typical temperature $T_{r} \lesssim 10^{9} \mathrm{GeV}$ which could be obtained neglecting the stage of parametric resonance [5]. On the other hand, such processes as baryon creation after inflation occur best of all outside the state of thermal equilibrium. Therefore, the stage of pre-heating may play an extremely important role in our cosmological scenario. Another consequence of the resonance effects is an almost instantaneous change of equation of state from the vacuum-like one to the equation of state of relativistic matter. 
This leads to suppression of the number of primordial black holes which could be produced after inflation.

L.K. was supported in part by the Canadian Institute for Advance Research cosmology program, and CITA. A.L. was supported in part by NSF grant PHY-8612280. A.S. is grateful to Profs. Y. Nagaoka and J. Yokoyama for their hospitality at the Yukawa Institute for Theoretical Physics, Kyoto University. A.S. was supported in part by the Russian Foundation for Basic Research, Project Code 93-02-3631, and by Russian Research Project "Cosmomicrophysics".

\section{References}

[1] A.D. Linde, Particle Physics and Inflationary Cosmology (Harwood, Chur, Switzerland, 1990).

[2] A.D. Dolgov and A.D. Linde, Phys. Lett. 116B, 329 (1982); L.F. Abbott, E. Fahri and M. Wise, Phys. Lett. 117B, 29 (1982).

[3] A.A. Starobinsky, in: Quantum Gravity, Proc. of the Second Seminar "Quantum Theory of Gravity" (Moscow, 13-15 Oct. 1981), eds. M.A. Markov and P.C. West (Plenum, New York, 1984), p. 103.

[4] A.D. Dolgov and D.P. Kirilova, Sov. Nucl. Phys., 51, 273 (1990); J. Traschen and R. Brandenberger, Phys. Rev. D 42, 2491 (1990).

[5] L.A. Kofman, A.D. Linde and A.A. Starobinsky, in preparation.

[6] Some authors discussed a possibility to add additional terms of the type of $\Gamma \dot{\phi}$ to the equation of motion in order to describe energy transfer due to particle creation. There was a hope that such terms may lead to a longer stage of inflation. We have found that this prescription does not correctly describe particle creation at the stage preceding a stage of oscillations of the scalar field, and that it is not possible to support inflation by the term $\Gamma \dot{\phi}[5]$.

[7] It is interesting that the rapid decay of the field $\phi$ can occur even if the field $\chi$ has a bare mass $m_{\chi}(0)$ much greater than the frequency of oscillations $m_{\phi}$. This rather unexpected regime is possible if the amplitude of oscillations is sufficiently large, $g \Phi \gg m_{\chi}^{2} / m_{\phi}$.

\section{Figure Caption:}

Fig. 1. The sketch of the stability/instability chart of the canonical Mathieu equation (3). 
This figure "fig1-1.png" is available in "png" format from: http://arXiv.org/ps/hep-th/9405187v2 